\title{
Single Particle Nanomechanics in Operando Batteries via Lensless Strain Mapping
}

Andrew Ulvestad, ${ }^{* \dagger}{ }^{\dagger}$ Andrej Singer, ${ }^{\dagger}$ Hyung-Man Cho, ${ }^{\ddagger}$ Jesse N. Clark, ${ }^{\S, \|}$ Ross Harder, ${ }^{\perp}$ Jorg Maser, $^{\perp}$ Ying Shirley Meng, and Oleg G. Shpyrko ${ }^{\dagger}$

${ }^{\dagger}$ Department of Physics, University of California-San Diego, La Jolla, California 92093-0319, United States

${ }^{\ddagger}$ Department of NanoEngineering, University of California-San Diego, La Jolla, California 92093-0448, United States

${ }^{\S}$ Stanford PULSE Institute, SLAC National Accelerator Laboratory, Menlo Park, California 94025, United States

"Center for Free-Electron Laser Science (CFEL), Deutsches Elektronensynchrotron (DESY), Notkestrasse 85, 22607 Hamburg, Germany

${ }^{\perp}$ Advanced Photon Source, Argonne National Laboratory, Argonne, Illinois 60439, United States

\section{Supporting Information}

ABSTRACT: We reveal three-dimensional strain evolution in situ of a single $\mathrm{LiNi}_{0.5} \mathrm{Mn}_{1.5} \mathrm{O}_{4}$ nanoparticle in a coin cell battery under operando conditions during charge/discharge cycles with coherent X-ray diffractive imaging. We report direct observation of both stripe morphologies and coherency strain at the nanoscale. Our results suggest the critical size for stripe formation is $50 \mathrm{~nm}$. Surprisingly, the single nanoparticle elastic energy landscape, which we map with femtojoule precision, depends on charge versus discharge, indicating hysteresis at the single particle level. This approach opens a powerful new avenue for studying battery nanomechanics, phase transformations, and capacity fade under operando conditions at the single particle level that will enable profound insight into the nanoscale mechanisms that govern electrochemical energy storage systems.
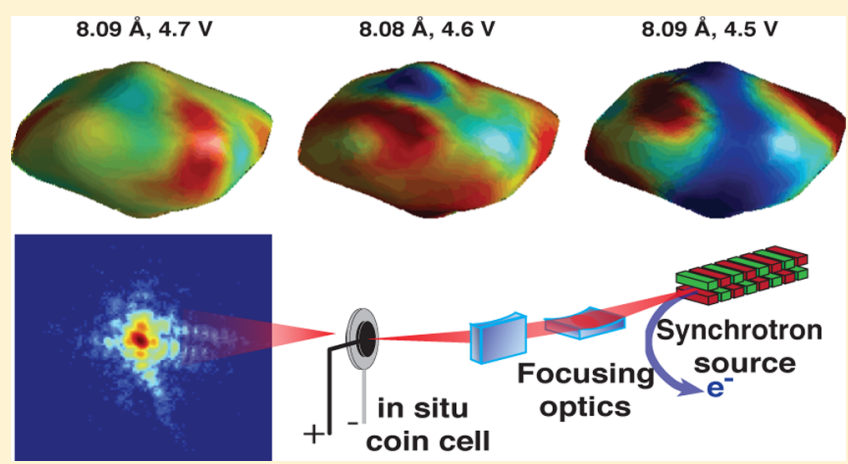

KEYWORDS: Battery, Operando, In Situ, Coherent X-ray Diffractive Imaging, Strain, LNMO

$\mathrm{L}$ ithium ion batteries are ubiquitous in mobile devices, ${ }^{1}$ increasingly used in transportation, ${ }^{2}$ and promising candidates for renewable energy integration into the electrical grid, ${ }^{3}$ provided the degradation of electrochemical performance upon use can be understood, mitigated, and ideally eliminated. ${ }^{4}$ Central to degradation mechanisms in nanostructured electrodes, which are increasingly used in batteries due to their enhanced functionality, are the nanomechanics of lithium ions, which remains insufficiently characterized at the single particle level under operando conditions. ${ }^{5,6}$ In particular, nanostructured spinel materials such as disordered $\mathrm{LiNi}_{0.5} \mathrm{Mn}_{1.5} \mathrm{O}_{4}$ (LNMO) are appealing as high voltage, high capacity, environmentally friendly, and low cost cathodes for use in numerous markets. ${ }^{7}$ However, capacity loss due to degradation is limiting its current use. Important degradation processes, including active material cracking, disconnection, and impedance increase can be understood in terms of strain evolution at the single particle level. Strain needs to be imagined in situ under operando conditions in order to provide insight into real processes and mechanisms. $^{8}$

Thermodynamic considerations characterize the type of strain induced in particular sections of the voltage profile. ${ }^{9} \mathrm{~A}$ composition-dependent voltage indicates a solid solution regime in which (de)insertion kinetics induce strain, which is an indirect probe of lithium concentration. ${ }^{10}$ Flat, or composition-independent, voltage profiles typically indicate two-phase coexistence, ${ }^{9}$ which induces strain required to maintain coherent (or semicoherent) interfaces between the phases, known as coherency strain. ${ }^{11}$ Several key material properties can be derived from both the number and width of coherent interfaces formed in a single particle, ${ }^{12}$ although there is some debate as to the mechanics of two-phase coexistence in several important materials (e.g., $\mathrm{LiFePO}_{4}$ ) due to the high elastic energy required. ${ }^{13}$

Elastic energy is useful in describing structural two-phase coexistence in battery materials, which is key to understanding degradation due to damage induced by the lattice mismatch.,14 The strain generated during, for example, the cubic-tetragonal phase transformation in $\mathrm{LiM}_{2} \mathrm{O}_{4}$ causes irreversible damage, including defect nucleation, which leads to large capacity fade. ${ }^{4}$ Structural transformations can be understood by mapping the elastic energy landscape, that is, the barrier height and width

Received: May 19, 2014

Revised: July 31, 2014

Published: August 20, 2014 

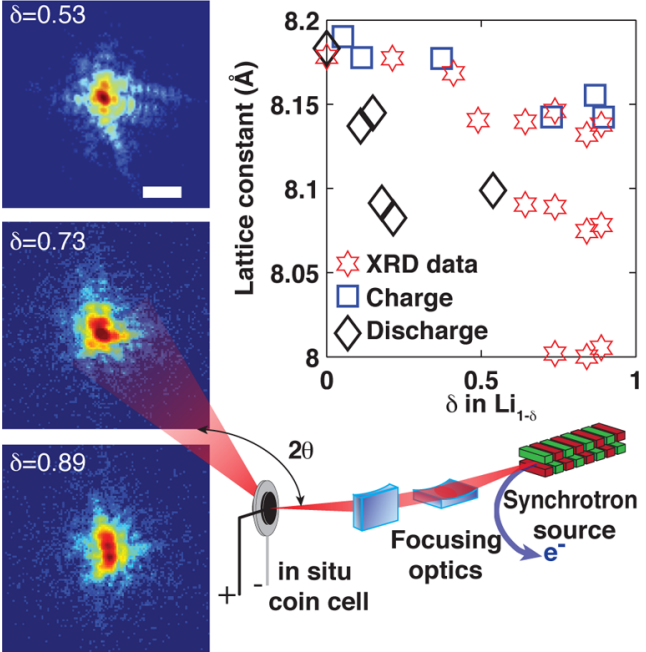

Figure 1. Experimental schematic of the in situ CXDI setup with lattice constant evolution inset. Diamonds and squares show lattice evolution during discharge and charge, respectively. Stars show ex situ $\mathrm{X}$-ray diffraction data during charge. Errors are within the symbols. The scale bar for diffraction data is $0.05 \mathrm{~nm}^{-1}$.

between the two energy minima. This two-state formalism is ubiquitous and very successful in describing diverse phenomena including formation of ferromagnetic and ferroelectric domains, ${ }^{95}$ spinodal decomposition, ${ }^{11}$ early universe scenarios, ${ }^{16}$ and simple molecules. ${ }^{17}$ Applied to batteries, it could suggest avenues to mitigate phase transformation induced damage.

Nanoscale strain measurement is thus useful in mapping lithium inhomogeneity, determining key material properties, and discerning the energy landscape, provided the full threedimensional information is known. Coherent X-ray diffraction imaging (CXDI) in Bragg geometry is a powerful tool that can provide this strain information at the nanoscale by utilizing interference from coherent X-rays coupled with phase retrieval algorithms to reconstruct the electron density and out of equilibrium displacement of nanocrystals. ${ }^{18-21}$ Recently, we used CXDI to map strain in pristine LNMO cathode particles and discovered inhomogeneous strain distributions that can be explained by a competition between various effects. ${ }^{10}$

In this Letter, we elevate CXDI to in situ, operando conditions to study the spatial and temporal strain evolution of a single nanoparticle in a LNMO cathode over the entire voltage profile during (dis)charge. Upon charging, solid solution exists for high lithium content, whereas multiple cubic phases coexist for low lithium content. ${ }^{22}$ The quantitative impact of each regime on the strain is largely unknown, and the fundamental lithiation mechanism (i.e., core/shell or phase field) is unresolved. Additionally, the spatial and temporal kinetics of the cubic-to-cubic phase transformation are largely unknown. Thus, this material displays phenomena pertinent to many promising batteries.

The experimental setup is depicted in Figure 1. Focused coherent X-rays are incident on an in situ coin cell (Supporting Information Figure S1) and the signal scattered by an individual LNMO particle satisfying the Bragg condition is recorded at the detector. The central location of the Bragg peak defines the average lattice constant of the single particle while the asymmetry indicates the strain. Both change in response to Lithium concentration. ${ }^{19}$ Lattice evolution during charge (squares) is consistent with our ex situ X-ray diffraction (XRD) measurement (red stars) taken during charge and literature values. ${ }^{22} \mathrm{We}$ will denote the $\alpha, \beta$, and $\gamma$ phase as the phases with $8.15,8.1$, and $8.0 \AA$ lattice constants, respectively, all of which are cubic (Supporting Information Figure S2). During discharge (diamonds) the single particle lattice shows a different behavior compared to XRD data for the $\beta$ phase, which is likely due to the decrease in diffusivity at low lithiation and hysteresis between charging and discharging. ${ }^{23}$

From the coherent diffraction data, we reconstruct the threedimensional displacement field $\mathrm{u}_{111}$ in an individual cathode particle with $40 \mathrm{~nm}$ resolution as defined by the phase retrieval transfer function (Supporting Information Figure S3). The conversion from phase to displacement for each charge state uses the average lattice constant of the particle at that charge state. In the two-phase regions, the initial phase is the reference state. Figure 2 displays the compressive (blue) and tensile (red) strain $\left(\partial_{x_{111}} u_{111}\right)$ evolution on the shell and core as the battery underwent the first discharge at a $\mathrm{C} / 2$ rate $(2 \mathrm{~h}$ for full discharge, see Supporting Information Figure S4 for electro-

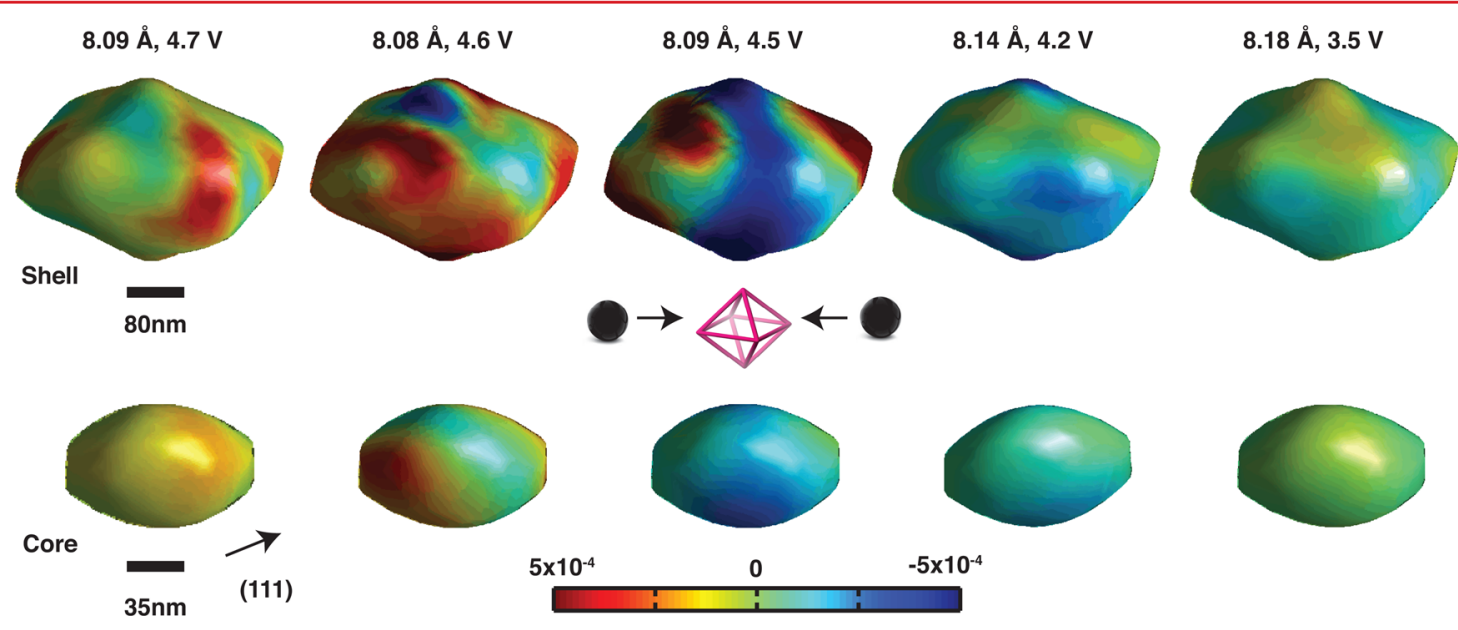

Figure 2. Isosurface projections of strain evolution. The nanoparticle shell and core both show inhomogeneous strain during discharge. Images are labeled by their respective lattice constant values and open circuit voltages. The highest lattice strain occurs immediately prior to the phase transformation. 

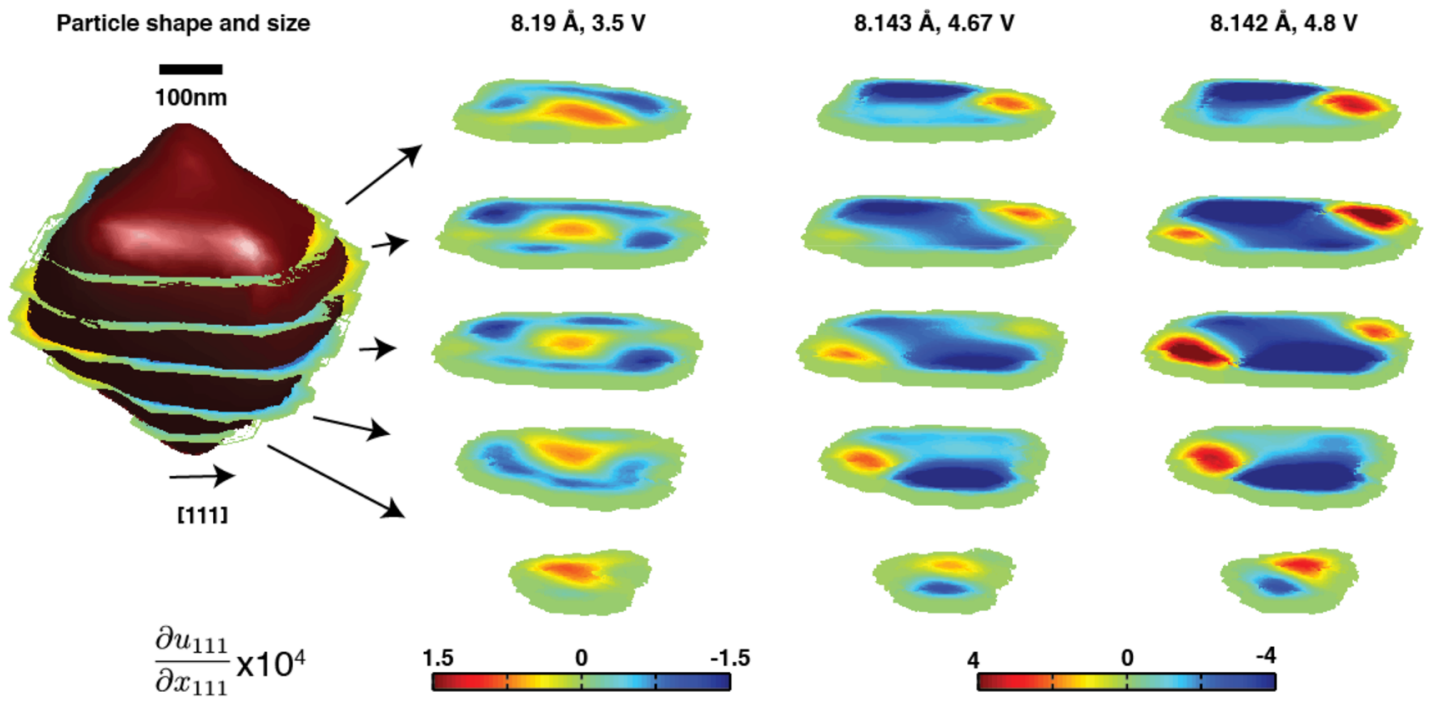

Figure 3. Interior strain distribution on selected cross sections at positions shown by the leftmost figure. Single-particle strain cross sections show the onset of coherency strain and resulting stripe patterns at 8.143 and $8.142 \AA$. Note the first slicing is scaled differently than the other two. Blue and red represent the $\alpha$ and $\beta$ phases, respectively, for the cross sections at 8.143 and $8.142 \AA$.

chemical data). The [111] direction is indicated. A schematic indicates that discharge corresponds to lithium insertion. The particle is octahedral in shape and roughly $400 \mathrm{~nm}$ in diameter, which is a size and shape commonly observed using scanning electron microscopy (Supporting Information Figure S5). We use our coherent powder diffraction study (Supporting Information Figure S6) to ensure the particle is representative of the average and stable. Effects due to X-ray exposure were confirmed to be negligible by repeated measurements. Note that the voltage is a global measurement, whereas the particle lattice constant is a single particle measurement.

The strain inhomogeneity in Figure 2 is striking. Early in the discharge cycle $(4.7 \mathrm{~V}, 8.09 \AA)$, strain manifests itself on the surface in the form of domain-like structures. State $2(4.6 \mathrm{~V}$, $8.08 \AA$ ) shows the onset of coherency strain, which we expect due to the flat voltage profile and phase coexistence in this region. State $3(4.5 \mathrm{~V}, 8.09 \AA)$ shows the strain builds as the particle nears the structural phase transformation, and then finally relaxes after the phase transformation $(4.2 \mathrm{~V}, 8.14 \AA$ A). Xray diffraction data (Figure 1 ) suggests that the material at 8.14 $\AA(4.2 \mathrm{~V})$ and $8.18 \AA(3.5 \mathrm{~V})$ is in the solid solution regime, where compressive/tensile strain correlates to lithium concentration. ${ }^{10}$ The strain is clearly inhomogeneous, indicating that phase field models of lithiation ${ }^{24}$ are more applicable than core-shell models, ${ }^{25}$ despite the $3 \mathrm{D}$ diffusion pathways in this structure. Strain is minimized at full lithiation ( $8.18 \AA$, $3.5 \mathrm{~V})$, as all unit cells are in principle equivalent at this point.

Full 3D strain evolution inside the particle during charging is shown in Figure 3 and quite revealing. Beginning with $8.19 \AA$ $(3.5 \mathrm{~V})$, we observe competition between pristine state strain and compressive strain at the edges of the particle due to the geometric effect described by the Young-Laplace model. ${ }^{26}$ During charge, both $8.143 \AA$ (4.67 V) and $8.142 \AA(4.8 \mathrm{~V})$ are in the multiple phase regions of the lattice constant data (Figure 1), which indicates two-phase coexistence and, thus, coherency strain. Two-phase coexistence is confirmed at the single particle level by an in situ powder diffraction study (Supporting Information Figure S6). To interpret phase separation, we apply the theory developed by Cahn and Hilliard. ${ }^{11}$ The free energy of a nonuniform binary solution is

$$
F=N_{\mathrm{V}} \int\left(f_{0}(c)+\kappa(\nabla c)^{2}+\frac{1}{2} \sigma_{i j} \epsilon_{i j}\right) \mathrm{d} V
$$

where the local lithium ion concentration, $c$, is the order parameter of the phase field model, $N_{\mathrm{V}}$ is the number of molecules per unit volume, and $f_{0}(c)=\Omega c(1-c)+k T(c \log c$ $+(1-c) \log (1-c))$ where $\Omega, k$, and $T$ represent the regular solution parameter, Boltzmann constant, and temperature, respectively. The first and second part of $f_{0}(c)$ are the enthalpic and entropic contributions that favor phase separation and phase mixing, respectively. The second term in eq 1 represents the "gradient energy" with coefficient $\kappa$. The final term is the sum of the product of the stress and strain tensors, which is the elastic energy. Both the gradient and elastic energy penalize spatial concentration modulation. An initially homogeneous, marginally stable mixture governed by eq 1 phase separates under perturbations, despite coherency strain, into a striped morphology that is preferred due to elastic energy relaxation at the particle boundaries. ${ }^{12,27}$

The width of the stripes (see Figure 3, 8.143 $\AA$ (4.67 V) and $8.142 \AA(4.8 \mathrm{~V}))$ can be related to the interfacial energy by a scaling relation ${ }^{12}$ derived from minimization of eq 1

$$
\lambda=2 w=\sqrt{\frac{2 \gamma L_{\mathrm{c}}}{\Delta f}} \quad \gamma \sim 106 \mathrm{~mJ} / \mathrm{m}^{2}
$$

Here, $\lambda$ is the period of the striping, $\gamma$ is the interfacial energy, $L_{\mathrm{c}}$ is the width of the particle along which the phase separation occurs, and $\Delta f$ is the difference in free energy density between the homogeneous and coherent phase-separated state. This interfacial energy is similar to $\mathrm{LiFePO}_{4}^{12,28}$ and roughly equivalent to the surface tension of water. The diffuse width of the stripe boundary, estimated from the images as $50 \mathrm{~nm}$, provides an estimate for the minimum size for two-phase coexistence. ${ }^{12}$ Particles below this size should not phase separate but exist entirely as one phase or the other. The width of both the stripes and the interface, thus, reveal significant information about this LNMO particle.

We also map the elastic energy landscape by utilizing the full three-dimensional strain distribution to evaluate the elastic energy which, under isotropic shear-free conditions, ${ }^{11}$ is 


$$
E_{\mathrm{s}}=\int \frac{1}{2} \Sigma_{i j} \sigma_{i j} \epsilon_{i j} \mathrm{~d} V=\frac{2 G+3 I}{2} \int\left(\frac{\partial u_{111}}{\partial x_{111}}\right)^{2} \mathrm{~d} V
$$

where $G$ and $I$ are the Lame constants for the material, estimated using molecular dynamics simulations of $\mathrm{LiMn}_{2} \mathrm{O}_{4}$ spinel, ${ }^{29,30}$ and the volume integral is over the entire particle. LNMO always maintains a cubic lattice structure (Figure 1 and Supporting Information Figure S2), so one strain component is sufficient to evaluate the sum. Elastic strain energy counts the strain due to deviations of the atoms from their equilibrium position, regardless of the underlying cause of the displacement. Figure 4 shows the values of the elastic energy, on the order of femtojoules, at different charge states.

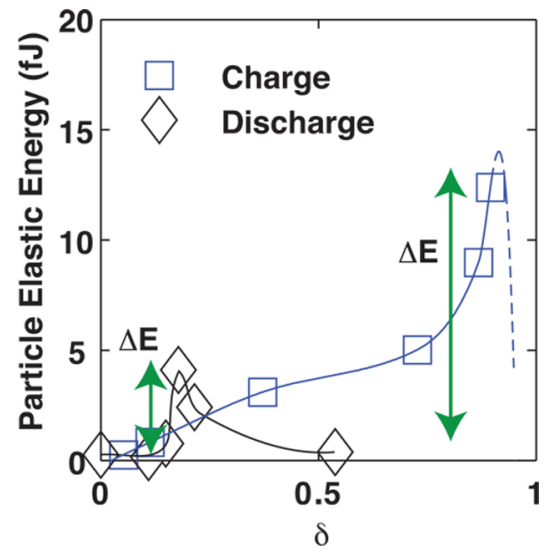

Figure 4. Elastic energy landscape of a single particle during charge and discharge. Uncertainties are within the symbols. Energy barriers to the phase transformation are indicated with green arrows. Dashed blue line is the expected shape after completion of the phase transformation.

The mapping of the energy landscape reveals surprising dynamics, including a clear difference in the location, energy, and asymmetry of the energy barrier between charge and discharge (confirmed for another particle, see Supporting Information Figure S7). Although hysteresis in a globally averaged variable, such as the voltage, is expected, this hysteresis is at the single particle level and involves the threedimensional strain field. It is unexpected and can perhaps be explained by accounting for losses in the form of irreversible elastic energy release via sound waves, cracks, and dislocation nucleation. ${ }^{28}$ Electrostatic repulsion may also play a role in determining the height of the energy barrier because it is much greater in the high $\delta$ vs low $\delta$ states as observed in diffusion coefficient measurements. ${ }^{23}$ Alternatively, the "uphill" diffusion required to form stripes can explain the decrease in effective diffusion coefficients. During discharge, we successfully pushed the particle from the $\beta$ phase to the $\alpha$ phase. During charge, we believe we were on the cusp of transforming from the $\alpha$ phase to either the $\beta$ or $\gamma$ phase due to the similarity in the strain distributions between the highest strain state during discharge and that during charge. The landscape indicates the phase transformation from large to small lattice constant is much worse in terms of elastic energy per unit cell than the reverse transformation, which must be included in modeling. The map suggests that focusing on ways to minimize the elastic energy, especially upon charge, for example, via the creation of more stripes by decreasing the interfacial energy in some way, is of paramount importance in increasing capacity retention and lifetime of LNMO spinel materials.

We studied strain evolution in situ at the single particle level under operando conditions during (dis)charging using CXDI. We discovered a surprisingly rich set of phenomena related to strain formation and propagation, coherency strain and striping, and the evolution of the elastic energy landscape with $40 \mathrm{~nm}$ spatial resolution and 0.5 femtojoule energy resolution. Going beyond traditional imaging, we used the strain mapping to determine key material properties, including the minimum size for two-phase coexistence and the interfacial energy, and we mapped the asymmetric energy barrier to the structural phase transformation. This approach unlocks a new, powerful way to conduct in situ studies under operando conditions of nanomechanics in many electrochemical energy storage systems at the single particle level.

\section{ASSOCIATED CONTENT}

\section{Supporting Information}

Sample synthesis, experimental method, and phase retrieval algorithms are described in additional detail. Eight additional figures are provided detailing sample characterization, phase retrieval, and an additional particle. This material is available free of charge via the Internet at http://pubs.acs.org.

\section{AUTHOR INFORMATION}

\section{Corresponding Author}

*E-mail: aulvesta@ucsd.edu.

\section{Author Contributions}

A.U. conducted the experiment, performed the data analysis, and wrote the paper. A.S. aided substantially in interpreting the data and editing the paper. H.M.C. aided the experiment and prepared the samples. J.C. wrote the phase retrieval code. R.H. aided the experiment. Y.S.M. and O.G.S. conceived of the experiment. All authors revised the manuscript.

\section{Notes}

The authors declare no competing financial interest.

\section{ACKNOWLEDGMENTS}

This work was supported by U. S. Department of Energy, Office of Science, Office of Basic Energy Sciences, under Contract DE-SC0001805. H.M.C. and Y.S.M. acknowledge the financial support by U. S. Department of Energy, Office of Basic Energy Sciences, under Award Number DE-SC0002357. O.G.S. and Y.S.M. are grateful to the UCSD Chancellor's Interdisciplinary Collaborators Award that made this collaboration possible. Use of the Advanced Photon Source, an Office of Science User Facility operated for the U. S. Department of Energy (DOE) Office of Science by Argonne National Laboratory, was supported by the U.S. D.O.E. under Contract No. DE-AC02-06CH11357. A.U. thanks beamline scientist David Vine and staff at Argonne National Laboratory and the Advanced Photon Source for their support. J.N.C gratefully acknowledges financial support from the Volkswagen Foundation.

\section{ABBREVIATIONS}

LNMO, $\mathrm{LiNi}_{1 / 2} \mathrm{Mn}_{3 / 2} \mathrm{O}_{4}$; CCD, charge coupled device; OCV, open circuit voltage; XRD, X-ray diffraction; SEM, scanning electron microscopy; CXDI, coherent $\mathrm{X}$-ray diffractive imaging 


\section{REFERENCES}

(1) Tarascon, J.-M.; Armand, M. Nature 2001, 414, 359-367.

(2) Armand, M.; Tarascon, J.-M. Nature 2008, 451, 652-657.

(3) Dunn, B.; Kamath, H.; Tarascon, J.-M. Science 2011, 334, 928935.

(4) Aricò, A. S.; Bruce, P.; Scrosati, B.; Tarascon, J.-M.; van Schalkwijk, W. Nat. Mater. 2005, 4, 366-377.

(5) Balke, N.; Jesse, S.; Morozovska, a N.; Eliseev, E.; Chung, D. W.; Kim, Y.; Adamczyk, L.; García, R. E.; Dudney, N.; Kalinin, S. V. Nat. Nanotechnol. 2010, 5, 749-754.

(6) Zhu, J.; Lu, L.; Zeng, K. ACS Nano 2013, 7, 1666-1675.

(7) Deng, H.; Belharouak, I.; Cook, R. E.; Wu, H.; Sun, Y.-K.; Amine,

K. J. Electrochem. Soc. 2010, 157, A447-A452.

(8) Wang, C.-M.; Li, X.; Wang, Z.; Xu, W.; Liu, J.; Gao, F.; Kovarik, L.; Zhang, J.-G.; Howe, J.; Burton, D. J.; Liu, Z.; Xiao, X.; Thevuthasan, S.; Baer, D. R. Nano Lett. 2012, 12, 1624-1632.

(9) Huggins, R. A. Advanced Batteries: Materials Science Aspects; Springer: New York, 2008; p 474.

(10) Ulvestad, A.; Cho, H. M.; Harder, R.; Kim, J. W.; Dietze, S. H.; Fohtung, E.; Meng, Y. S.; Shpyrko, O. G. Appl. Phys. Lett. 2014, 073108, 2-6.

(11) Cahn, J. Acta Metall. 1961, 9, 795-801.

(12) Cogswell, D. A.; Bazant, M. Z. ACS Nano 2012, 6, 2215-2225.

(13) Delmas, C.; Maccario, M.; Croguennec, L.; Le Cras, F.; Weill, F. Nat. Mater. 2008, 7, 665-671.

(14) Sun, Y.-K.; Chen, Z.; Noh, H.-J.; Lee, D.-J.; Jung, H.-G.; Ren, Y.; Wang, S.; Yoon, C. S.; Myung, S.-T.; Amine, K. Nat. Mater. 2012, 11, 942-947.

(15) Shpyrko, O. G.; Isaacs, E. D.; Logan, J. M.; Feng, Y.; Aeppli, G.; Jaramillo, R.; Kim, H. C.; Rosenbaum, T. F.; Zschack, P.; Sprung, M.; Narayanan, S.; Sandy, A. R. Nature 2007, 447, 68-71.

(16) Ulvestad, A.; Albrecht, A. Creating universes with thick walls. Phys. Rev. D, 2012, 85.

(17) Frauenfelder, H.; Leeson, D. T. Nat. Struct. Biol. 1998, 5, 757759.

(18) Yang, W.; Huang, X.; Harder, R.; Clark, J. N.; Robinson, I. K.; Mao, H. Nat. Commun. 2013, 4, 1680.

(19) Robinson, I.; Harder, R. Nat. Mater. 2009, 8, 291-298.

(20) Pfeifer, M. a; Williams, G. J.; Vartanyants, I. a; Harder, R.; Robinson, I. K. Nature 2006, 442, 63-66.

(21) Clark, J. N.; Huang, X.; Harder, R.; Robinson, I. K. Nat. Commun. 2012, 3, 993-999.

(22) Kim, J.; Myung, S.; Yoon, C. S.; Kang, S. G.; Sun, Y. Chem. Mater. 2004, 7, 906-914.

(23) Yang, M.-C.; Xu, B.; Cheng, J.-H.; Pan, C.-J.; Hwang, B.-J.; Meng, Y. S. Chem. Mater. 2011, 23, 2832-2841.

(24) Singh, G. K.; Ceder, G.; Bazant, M. Z. Electrochim. Acta 2008, 53, 7599-7613.

(25) Christensen, J.; Newman, J. J. Electrochem. Soc. 2006, 153, A1019-A1030.

(26) Robinson, I. K. J. Phys. Soc. Jpn. 2012, 82, 1-7.

(27) Khachaturyan, A. G. Phys. Status Solidi 1969, 35, 119-132.

(28) Van der Ven, a.; Garikipati, K.; Kim, S.; Wagemaker, M. J. Electrochem. Soc. 2009, 156, A949-A957.

(29) Lee, S.; Park, J.; Sastry, a. M.; Lu, W. J. Electrochem. Soc. 2013, 160, A968-A972.

(30) Cho, H.-M.; Meng, Y. S. J. Electrochem. Soc. 2013, 160, A1482A1488. 